\title{
Quantification of ortholog losses in insects and vertebrates Stefan Wyder ${ }^{* \dagger}$, Evgenia V Kriventseva ${ }^{\ddagger \dagger}$, Reinhard Schröder ${ }^{\S}$, Tatsuhiko Kadowaki ${ }^{\llbracket}$ and Evgeny M Zdobnov*¥¥
}

\begin{abstract}
Addresses: *Department of Genetic Medicine and Development, University of Geneva Medical School, 1211 Geneva, Switzerland. ${ }^{+}$Swiss Institute of Bioinformatics, rue Michel-Servet, 1211 Geneva, Switzerland. *Department of Structural Biology and Bioinformatics, University of Geneva Medical School, rue Michel-Servet, 1211 Geneva, Switzerland. §Interf. Institut für Zellbiologie, Abt. Genetik der Tiere, Universität Tübingen, 72076 Tübingen, Germany. "Graduate School of Bioagricultural Sciences, Nagoya University, Chikusa, Nagoya 464-8601, Japan. YImperial College London, South Kensington Campus, London SW7 2AZ, UK.
\end{abstract}

Correspondence: Evgeny M Zdobnov. Email: evgeny.zdobnov@medecine.unige.ch

Published: 16 November 2007

Genome Biology 2007, 8:R242 (doi:10.1 I86/gb-2007-8-II-r242)

The electronic version of this article is the complete one and can be found online at http://genomebiology.com/2007/8/I I/R242
Received: 30 June 2007

Revised: 4 October 2007

Accepted: 16 November 2007

(c) 2007 Wyder et al.; licensee BioMed Central Ltd.

This is an open access article distributed under the terms of the Creative Commons Attribution License (http://creativecommons.org/licenses/by/2.0), which permits unrestricted use, distribution, and reproduction in any medium, provided the original work is properly cited.

\begin{abstract}
Background: The increasing number of sequenced insect and vertebrate genomes of variable divergence enables refined comparative analyses to quantify the major modes of animal genome evolution and allows tracing of gene genealogy (orthology) and pinpointing of gene extinctions (losses), which can reveal lineage-specific traits.
\end{abstract}

Results: To consistently quantify losses of orthologous groups of genes, we compared the gene repertoires of five vertebrates and five insects, including honeybee and Tribolium beetle, that represent insect orders outside the previously sequenced Diptera. We found hundreds of lost Urbilateria genes in each of the lineages and assessed their phylogenetic origin. The rate of losses correlates well with the species' rates of molecular evolution and radiation times, without distinction between insects and vertebrates, indicating their stochastic nature. Remarkably, this extends to the universal single-copy orthologs, losses of dozens of which have been tolerated in each species. Nevertheless, the propensity for loss differs substantially among genes, where roughly $20 \%$ of the orthologs have an 8-fold higher chance of becoming extinct. Extrapolation of our data also suggests that the Urbilateria genome contained more than 7,000 genes.

Conclusion: Our results indicate that the seemingly higher number of observed gene losses in insects can be explained by their two- to three-fold higher evolutionary rate. Despite the profound effect of many losses on cellular machinery, overall, they seem to be guided by neutral evolution.

\section{Background}

The evolution of gene repertoires is mostly driven by gene duplications and gene losses. Duplications can arise by shortrange copying of individual genes or of longer multigene DNA segments, or even result from whole genome duplications $[1,2]$. Copies of single genes are frequently associated with retrotransposition [3], whereas unequal homologous recombination copies DNA segments of varying length. Gene proliferation, on the other hand, is balanced by gene losses, either through acquiring deleterious mutations that eventually disable the genes or as a consequence of unequal homologous recombination. 
Massive gene losses of olfactory receptors were reported in human and ape families compared to mice [4], which have been speculated to be linked with the acquisition of full trichromatic vision, lowering the demand for olfaction [5]. Ohlson's 'less-is-more' hypothesis emphasizes that loss-offunction mutations may play a beneficial role in evolution [6]; an example for adaptive gene loss is the near-complete fixation of a null allele of CASP12 in the human lineage [7], presumably to confer protection from severe sepsis. Gene losses have also been implicated in reproductive isolation of Drosophila races [8].

The fast growing number of available vertebrate and insect genomes allows increasingly refined comparisons and the quantification of the major modes of animal genome evolution. The recent sequencing of the honeybee [9] and the Tribolium beetle [10] genomes extends insect genomics from only Dipteran species to the orders of Hymenoptera and Coleoptera, which radiated about 300 million years ago [11]. This allowed us to quantify and date losses of orthologous groups across ten bilaterian species in the first analysis that consistently compares five insects (phylum Arthropoda) and five vertebrates (phylum Chordata). Previous studies of gene losses have been focussed on mammals [12], vertebrates [13], or included only a single insect [14], or two dipterans [15]. Reassuringly, our analysis recovered previously published cases of gene losses, such as the loss of DNA methylation [16], and the heavy rearrangement of the circadian clock [17] in Diptera.

\section{Results}

\section{Quantification of ortholog losses}

To systematically identify gene losses in vertebrate and insect representatives of Bilaterian species, we delineated orthologous groups based on all-against-all Smith-Waterman comparisons using the official gene sets of five vertebrates (human, mouse, opossum, chicken and pufferfish) and five insects (fruitfly, malaria mosquito, dengue/yellow fever mosquito, honeybee and red flour beetle) (see details in Materials and methods). The species choice aimed to maximize the phylogenetic coverage with similar lineage radiation times in both deuterostomia and protostomia. The fraction of genes with recognized orthology among these species represents about $70-80 \%$ of their predicted gene pools. The comparative analysis of the shared content of gene repertoires across these species is discussed in the study presenting the analysis of the first beetle genome, that of Tribolium castaneum [10], and here we focus on the analysis of losses of orthologous genes. According to their phyletic distribution and gene copynumber in each of the species, we considered the following types of orthologous groups reflecting different selection pressures: the universal single-copy orthologs (U); the universal multiple-copy orthologs $(\mathrm{N})$; patchy orthologs $(\mathrm{P})$ that are present in both phyla in at least three species, in single or multiple copies; and insect- or vertebrate-specific orthologs (I and $\mathrm{V}$, respectively). While universal single-copy orthologs (U) are evolving under a distinct pressure for copy-number control, the number of universal multiple-copy orthologs (N) under similarly strict copy-number control is extremely low (only six groups have equal multiple-copy gene number in at least nine species). Although $\mathrm{U}, \mathrm{N}$ and $\mathrm{P}$ orthologs must all have been present in the last common ancestor of insects and vertebrates, the Urbilateria, losses in these fractions occur at different rates.

Figure $1 \mathrm{~b}$ shows the size distribution of the orthologous fractions and the number of losses in each species and ortholog category. The tree shown in Figure 1a illustrates the species phylogeny, which allowed us to infer the number of losses on the internal branches, assuming evolutionary parsimony, which minimizes the number of events required to explain the phyletic gene distribution in each orthologous group. The phylogenetic tree was reconstructed using maximum-likelihood analysis of the concatenated alignment of 1,150 universal single-copy orthologs [10] where the lengths of the branches are proportional to the number of accumulated mutations, allowing us to compare the gene loss rates with the rates of lineage divergence (measured as the rate of protein substitutions). For the branches closest to the root, the numbers of gene losses cannot be inferred without an additional outgroup.

The analysis identified hundreds of differentially lost Urbilaterian genes of $\mathrm{U}, \mathrm{N}$ and $\mathrm{P}$ orthologs in each of the species (see table of Figure 1). Overall, about $40 \%$ of ancient orthologous groups have been lost in at least one (out of the ten) species, illustrating the extent of the evolutionary flexibility of gene pools. Moreover, there are dozens of genes lost in each of the species that otherwise appear as universal single-copy orthologs. Koonin et al. [14] noted that nearly all pan-eukaryotic single-copy orthologs are subunits of known protein complexes; nevertheless, the observed losses indicate that even seemingly tightly constrained genes are, to a certain degree, dispensable in evolution.

\section{Number of losses correlate with molecular divergence} The inferred distribution of losses over the internal branches of the species phylogeny allowed us to correlate them with the estimated geological time of species radiations and the molecular evolutionary rate in each of the lineages. The molecular rates of evolution were quantified using genome-wide maximum-likelihood analysis of amino acid substitutions in the well aligned regions of single-copy orthologs (see Materials and methods). Figure 2a displays the correlation of the number of losses of the different types of orthologs plotted versus the protein sequence divergence. Losses of $\mathrm{U}$ and $\mathrm{N}$ orthologs (Figure 2b) occur only at the terminal branches as the fraction definition requires presence of the orthologous genes in at least nine species, whereas losses of $\mathrm{P}$ orthologs (Figure 2c) occur at both internal and terminal branches. Correlations are statistically significant for all categories (see 


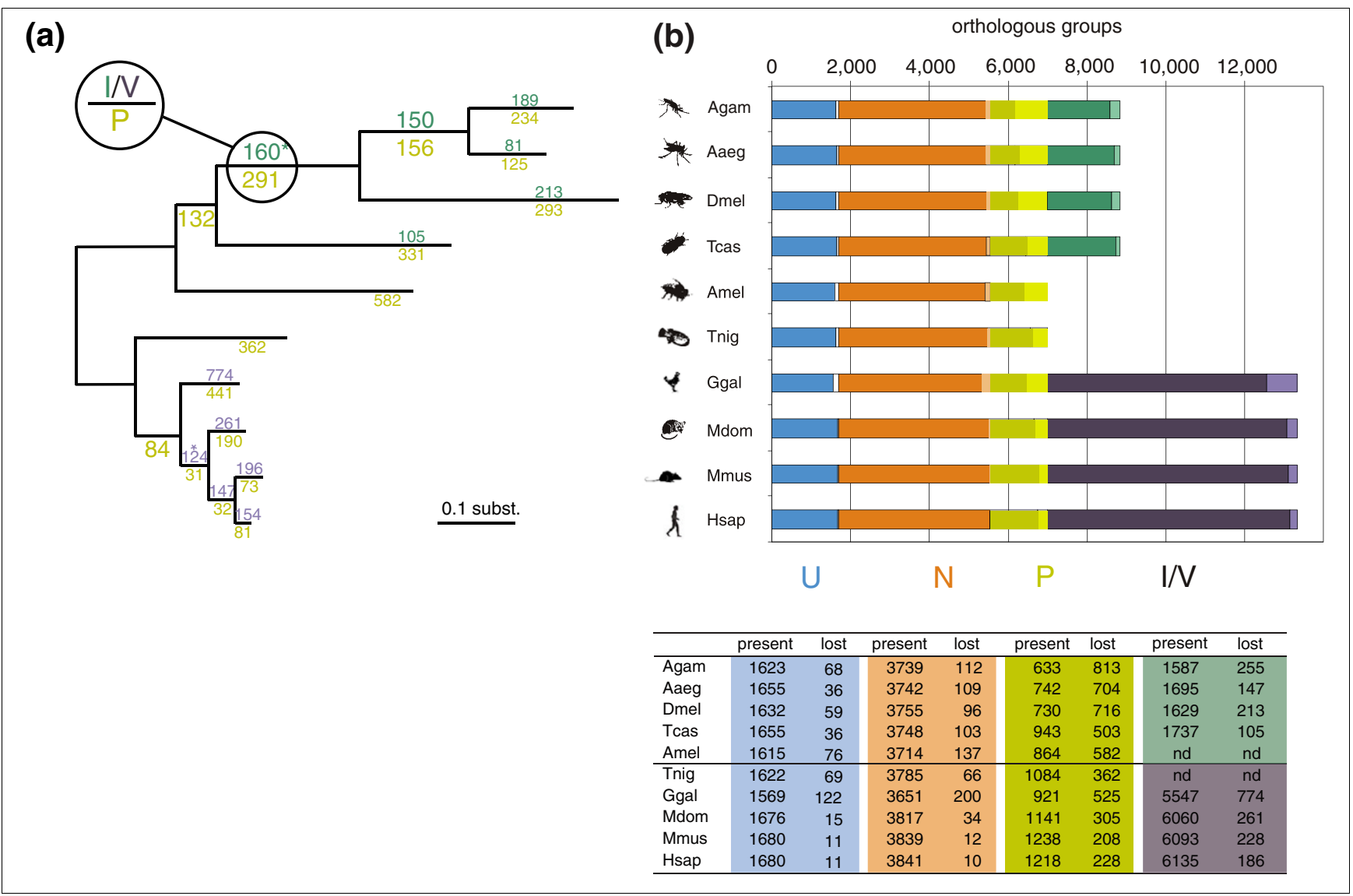

\section{Figure I}

Quantification of orthologous gene losses in insects and vertebrates. (a) The phylogenetic relations among the organisms are illustrated by the tree, with branch length proportional to the rate of amino acid substitutions estimated using the maximum-likelihood approach. The number of orthologous groups lost on the internal phylogenetic branches were inferred using the Dollo parsimony principle and are shown on the phylogenetic tree above branches for the I/V fraction, and below branches for the $P$ fraction. *The presence in two species was sufficient to infer losses of $I / V$ orthologous groups. (b) The number of orthologous group losses in the five main categories: $\mathrm{U}$, universal single-copy genes (blue, present in all species except the one in question); $\mathrm{N}$, universal multiple-copy genes (orange, present in at least nine species); P, patchy orthologs (yellow, present in both phyla in at least three species, in one or multiple copies); I/V , insect- or vertebrate-specific orthologous groups (present only in insects (green) or vertebrates (violet), in at least three species. The dark parts of the bars depict the number of contemporarily present orthologous groups, and the light parts depict the number of inferred losses. AGAM, Anopheles gambiae; AAEG, Aedes aegypti; DMEL, Drosophila melanogaster; TCAS, Tribolium castaneum; AMEL, Apis meliferia; HSAP, Homo sapiens; MMUS, Mus musculus; MDOM, Monodelphis domestica; GGAL, Gallus gallus; TNIG, Tetraodon nigroviridis.

Figure 2 legend for details), and there is no distinction between insects and vertebrates. Moreover, although the absolute numbers of insect- and vertebrate-specific losses appear different, they in fact follow the same trend when normalized to the total number of the phylum-specific orthologous groups (Figure 2d; see Additional data file 1 for a graph with absolute numbers). The different slopes of the regression lines reflect the varying stringency of evolutionary constraints that differ between the postulated types of orthologs. Despite the difference in the absolute numbers of lost $U$ and $\mathrm{N}$ orthologs, their rates of loss are indistinguishable when normalized to the number of such orthologous groups, indicating the same level of purifying selection (Figure $2 b$ ). The data show that $\mathrm{P}$ orthologs are about 8-fold less constrained than $\mathrm{U}$ and $\mathrm{N}$ orthologs; this roughly corresponds to about $20 \%$ of the common Bilateria gene pool evolving 8 times faster than the remaining, more constrained fraction. I and V orthologs appear to be about three-fold more constrained than $\mathrm{P}$ orthologs, which is not surprising as they may represent a similar mixture of a slower evolving fraction of $80 \%$ and a faster evolving minority. The level of correlation between the number of losses and the protein sequence divergence rates (Figure 2) is similar to that observed between other genome-wide measures of species divergence [18]. Chicken was excluded from this and all following analyses as a clear outlier (see Discussion).

\section{Insects evolve two to three times faster than vertebrates}

Protein sequence divergence is significantly larger between insects than between vertebrates (see the longer branch lengths in Figure 1; Mann-Whitney $\mathrm{U}$ test, $p=0.009$ ). 


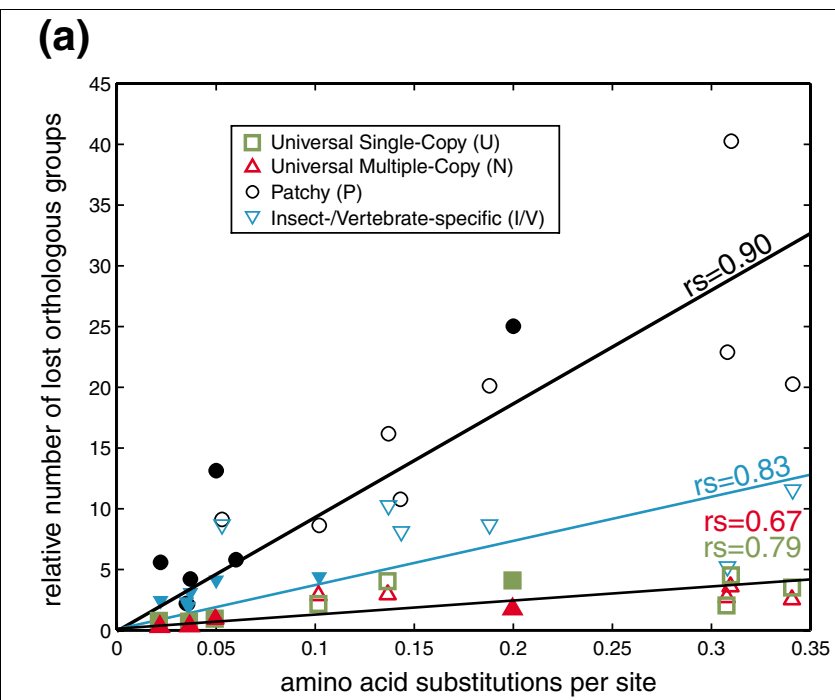

(b)

(c)
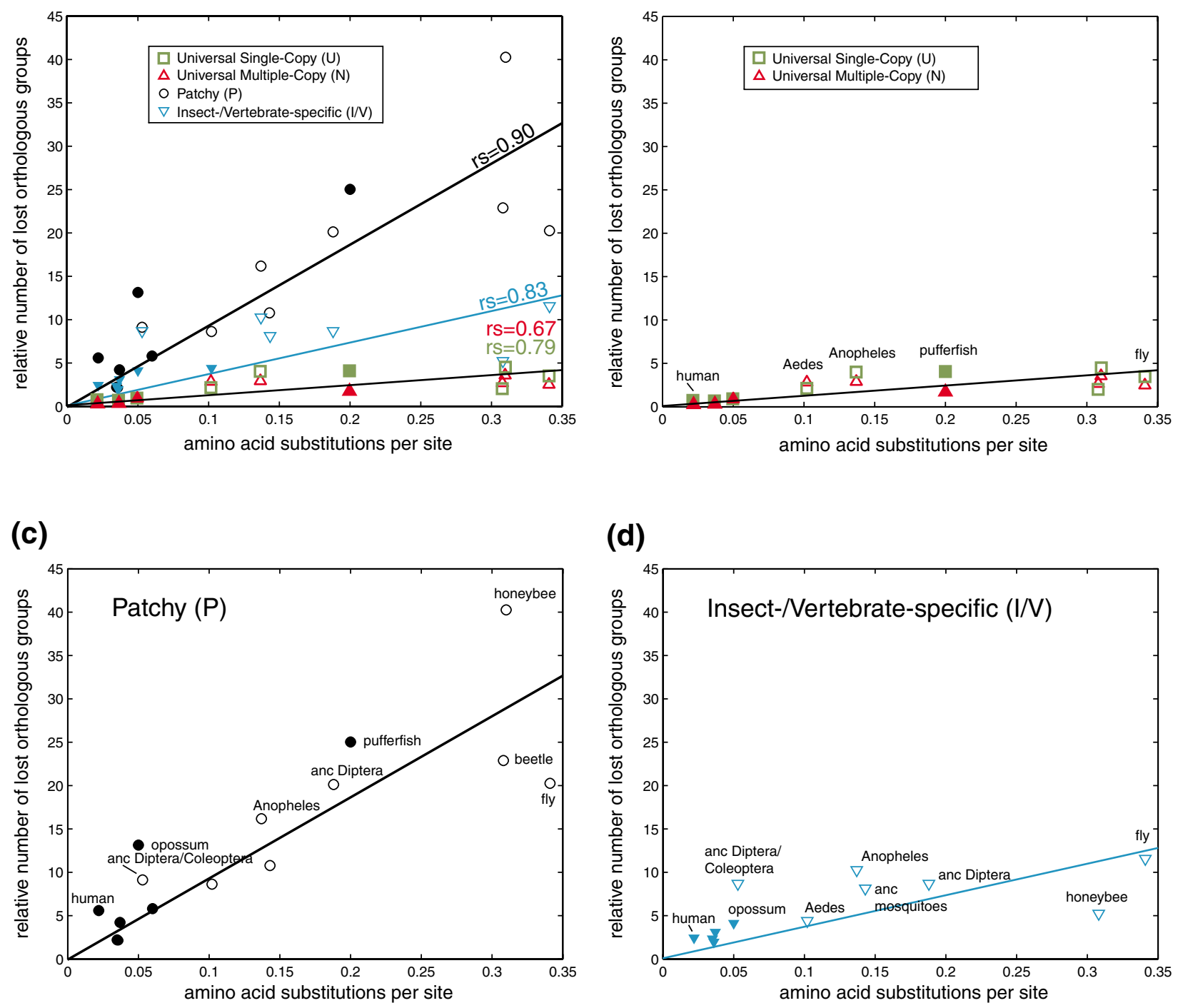

(d)

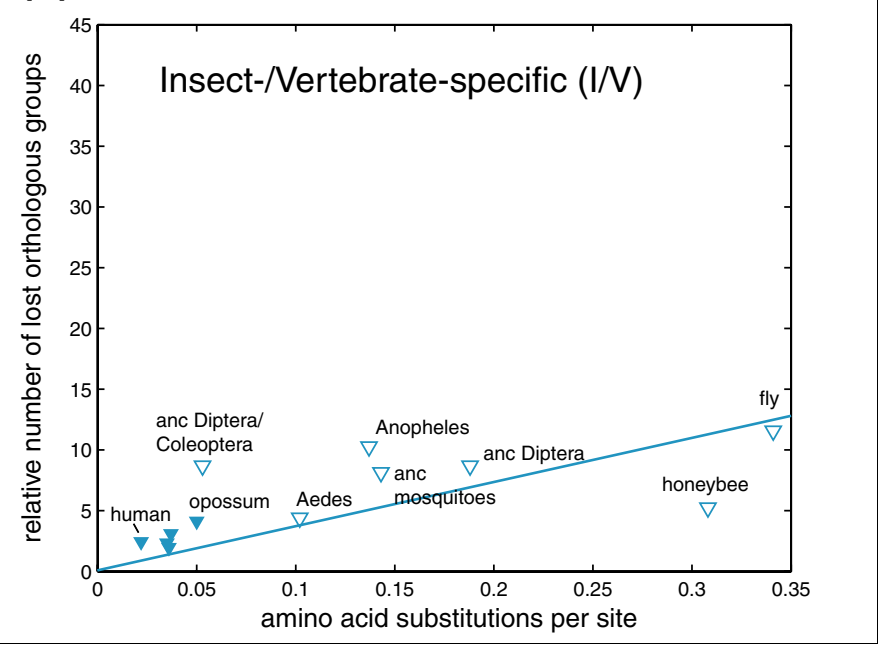

Figure 2

The number of ortholog losses correlates with the rate of amino acid substitutions. The number of orthologous group $(U, N, P, I / V)$ losses normalized with the total size of the fraction is plotted versus the branch length of the maximum-likelihood phylogenetic tree (Figure I). (a) All ortholog types combined; (b) $\cup$ and $N$ orthologs; (c) Patchy orthologs; (d) Insect- and vertebrate-specific orthologs. Filled symbols denote vertebrates and open symbols denote insects. Spearman rank correlations: $U$ orthologs, $r s=0.79, p=0.015 ; \mathrm{N}$ orthologs, $r s=0.67, p=0.05 ; \mathrm{P}$ orthologs, $r s=0.90, p<0.01 ; \mathrm{I} / \mathrm{V}$ orthologs, $r s=0.83, p<0.01$. Regression slopes for $U$ and $N$ are not statistically different. Anc, ancestral.

Similarly, this is reflected in the observation of significantly more frequent gene losses in insects than in vertebrates (Mann-Whitney U test: $\mathrm{N}$ orthologs, $p=0.016$; P orthologs, $p$ $=0.04)$. In comparison with vertebrates, the rate of evolution in bee and beetle is about two-fold higher and up to three-fold higher in Diptera. This especially high rate of evolution in Diptera, particularly at the base of the Dipteran radiation, has been noted previously [19].

\section{Lower estimate of the Urbilateria number of genes}

Despite inherent dating uncertainties, the correlation between the number of lost orthologous groups and diver- gence times is significant for $\mathrm{U}$ and $\mathrm{P}$ orthologs (Spearman rank correlations: $\mathrm{U}$ orthologs, $\mathrm{rs}=0.84, p=0.007 ; \mathrm{N}$ orthologs, $\mathrm{rs}=0.58, p=0.11 ; \mathrm{P}$ orthologs, $\mathrm{rs}=0.57, p=0.03$ ), indicating that losses of ancient genes occur in a roughly clock-like manner. The good correlation between the rate of losses with molecular rate and time indicates their stochastic nature. Projection of these trends as shown in Figure 3 to 600 million years ago (MYA), presumably dating the radiation of insects and vertebrates, suggests that over 1,000 (95\% confidence interval 799-1,456) Urbilaterian genes have been lost from insects and only half this number ( $95 \%$ confidence interval 404-678) from vertebrates. This leads to the lower 


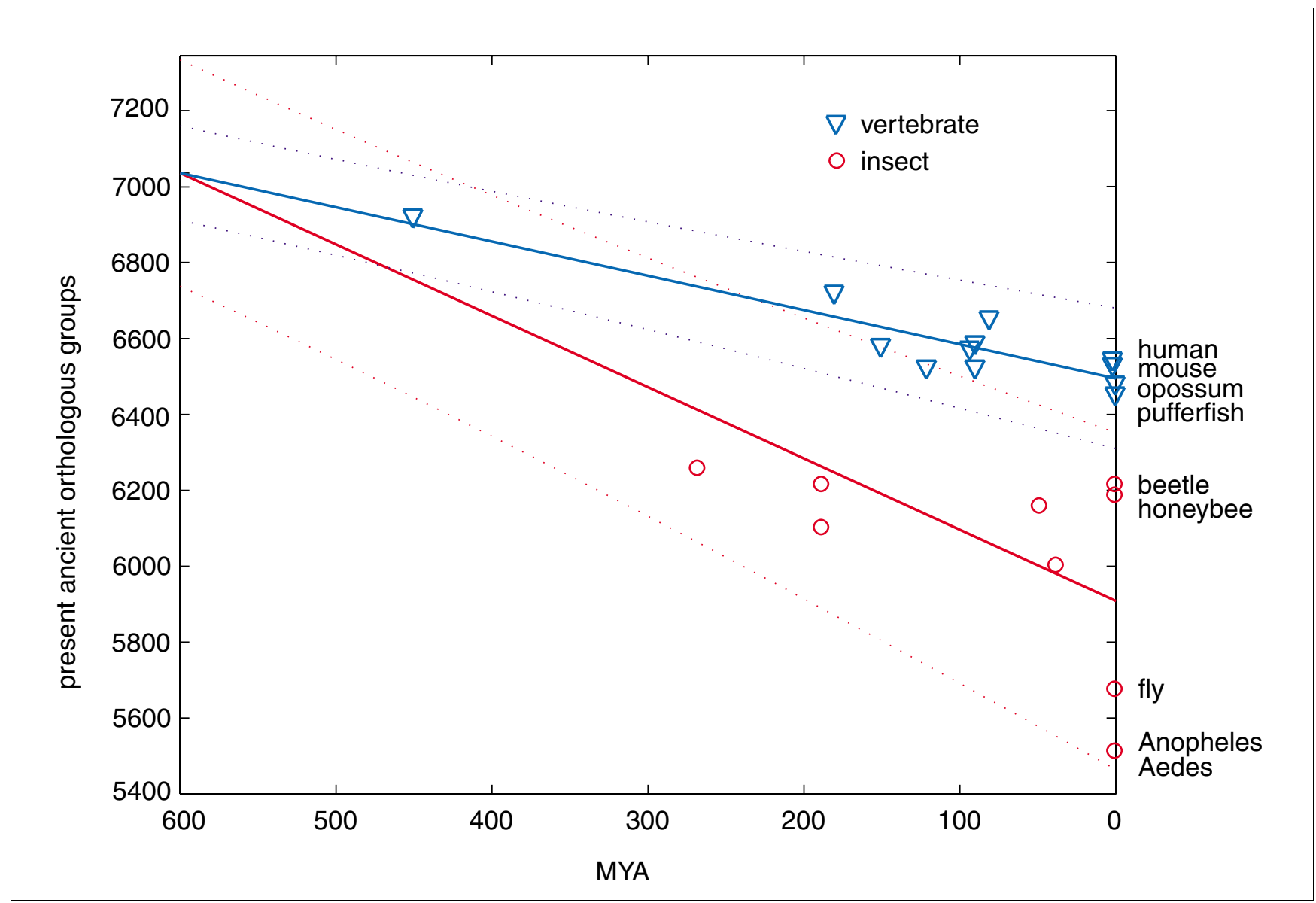

Figure 3

Extrapolation of number of ancient $(\mathrm{U}, \mathrm{N}$ and $\mathrm{P}$ ) orthologs to Urbilateria. The regression lines (and their $90 \%$ confidence intervals) are drawn using the number of $\mathrm{U}, \mathrm{N}$ and $\mathrm{P}$ orthologous groups in current species, the estimates for putative ancestors, including the inferred number of losses (Figure I), and the assumed split of insects and vertebrates about 600 MYA against the species radiation time. Remarkably, the naïve counting of orthologous groups that have at least one insect and at least one vertebrate member results in 7, I I 4 likely Urbilateria genes.

estimate of the number of Urbilaterian genes of just over 7,000 (remarkably, we obtained 7,114 orthologous groups with at least one insect and one vertebrate member). This estimate, however, does not take into account: genes that currently appear as insect- or vertebrate-specific, many of which could be of Urbilaterian origin; closely related Urbilateria paralogs that remain unresolved and are likely grouped together in N groups; as well as fractions of fast diverging genes that escaped our orthology classification.

\section{Functional load of losses}

Recovered known facts as positive controls

Reassuringly, closer inspection of several of the predicted cases of lost genes pointed to recently published findings of lineage-specific biology.

Hedgehog signaling pathway rearrangements in Drosophila Hedgehog signaling pathway rearrangements in Drosophila have been reported where orthologs of human Sil, Hip and
Gas1 are missing from Drosophila [20], and homologs of polaris/TG737 (nompB) and Kif3a (Klp64D) appear to have roles unrelated to hedgehog signaling $[21,22]$.

\section{Sid-1/tag- I 30 gene loss in all Diptera}

Sid-1/tag-13O genes have been lost in all Diptera but are present in bee and Tribolium as reported by Weinstock et al. [9]. Sid-1 is implicated in the cellular import of RNA interference signal and enables passive uptake of double-stranded RNA (yet, Sid-1 is likely to be a Caenorhabditis elegans invention as its inparalog, TAG13o, is less derived (Additional data file 2)).

\section{DNA-methyltransferases DNMTI and DNMT3B lost in the Coleoptera/Diptera ancestor}

DNA-methyltransferases DNMT1 and DNMT3B have been lost in the Coleoptera/Diptera ancestor, consistent with their loss reported in Diptera [23] and their surprising presence in honeybee $[9,16]$. 
Table I

\begin{tabular}{|c|c|c|c|c|c|}
\hline Pathway & Source & Genes & Total & $\begin{array}{l}\text { Only in } \\
\text { beetle }\end{array}$ & Significance \\
\hline Neuroactive ligand-receptor interaction & KEGG & NPFFRI, NPFFR2, BZRP, TSPO, GALRI, GALR2, GALR3 & 6 & 6 & 0.099 \\
\hline$A B C$ transporters - general & KEGG & $A B C A I, A B C A 4, A B C A / 2, A B C C 5, A B C C / 2$ & 5 & 3 & 4.73E-005* \\
\hline Oxidative phosphorylation & KEGG & ATP6V0E, UCRC (7.2 kDa), NDUFA7, COX7C & 4 & 4 & 0.04 \\
\hline Cell cycle & KEGG & CDC7, CCNEI, DBF4 & 3 & 3 & 0.12 \\
\hline Folate biosynthesis/starch and sucrose metabolism & KEGG & RAD54B, SETX & 2 & 2 & 0.06 \\
\hline Alkaloid biosynthesis II & KEGG & DDHDI, SLC27A2 & 2 & 0 & $0.02 \dagger$ \\
\hline Regulation of actin cytoskeleton & KEGG & FGDI, IQGAPI & 2 & 0 & \\
\hline Cholera - infection & KEGG & ATP6VOEI, TRIM23 & 2 & 1 & 0.06 \\
\hline Purine metabolism & KEGG & PDEIC, POLR2L & 2 & 2 & 0.49 \\
\hline Ribosome & KEGG & RPL29, RPL39 & 2 & 2 & 0.53 \\
\hline Neurodegenerative disorders & KEGG & $B C L 2 L I, N G F R$ & 2 & 0 & 0.06 \\
\hline Propanoate metabolism & KEGG & $M L Y C D, S L C 27 A 2$ & 2 & 0 & 0.07 \\
\hline Methionine metabolism & KEGG & DNMTI, DNMT3B & 2 & 0 & \\
\hline Oxidative stress induced gene expression via Nrf2 & Biocarta & HMOXI, NGFR & 2 & I & $0.02^{\dagger}$ \\
\hline
\end{tabular}

The statistical significance of the coordinated losses of at least two genes per pathway was calculated using hypergeometric test $\left(* p<0.01, t^{\dagger} p<0.05\right)$.

A candidate insect telomerase reverse transcriptase

A candidate insect telomerase reverse transcriptase (TERT) is present in honeybee and Tribolium but absent in all Dipterans. The absence of TERT in Diptera seems to be correlated with the loss of telomeric TTAGG repeats [24].

\section{Sterol metabolism, NAD biosynthesis, and other losses}

Sterol metabolism, NAD biosynthesis, and other losses proposed earlier from the comparison of the fly and the mosquito genomes [23] seem to have been lost in all insects sequenced so far, that is, before the appearance of holometabolous insects. Exceptions are a dihydroxyacetone kinase 1 lost from both Drosophila and Anopheles, a C-5 sterol desaturase and a histidine ammonia-lyase present in only Tribolium and two genes present in only honeybee, an ornithine carbamoyltransferase and a malonyl-CoA decarboxylase.

\section{Novel case stories}

Below we describe some of the identified losses that are likely to have had an impact on the functional divergence of the lineages, exemplifying losses of different types of orthologs, from the most conserved single-copy genes to orthologs with a highly patchy phylogenetic distribution. It has been suggested that secondary gene losses can be driven by the losses of key players of particular pathways or complexes that disable their functionality [25-27]. Hence, we mapped losses to the characterized biochemical pathways annotated for human orthologs; the results for Tribolium and its ancestor are overviewed in Table 1. However, having low numbers of losses per pathway [26], we concentrated more on providing examples of losses of directly interacting genes, reported for Drosophila from protein-protein interaction screens [28] and literature co-citations via human orthologs [29].
Losses of universal single-copy orthologs

An example of a universal single-copy ortholog missing in Drosophila is a $35 \mathrm{kDa}$ protein associated with U11 snRNPs. U11 and U12 are components of the minor spliceosome responsible for the splicing of a small number of U12-type introns ( $<1 \%$ in both humans and flies) [30]. The minor spliceosome is widely conserved from plants to humans, including most insects but absent from C. elegans. Lack of a clear ortholog of U11 snRNA and the associated $35 \mathrm{kDa}$ protein has been initially proposed [31], but Schneider et al. [32] identified a highly divergent U11 snRNA. The $31 \mathrm{kDa}$ and $35 \mathrm{kDa}$ proteins seem to be missing from all Drosophila species and a $25 \mathrm{kDa}$ protein is absent from Diptera [33]. Interestingly, the loss of U11/U12 spliceosomal proteins in Drosophila is accompanied by the loss of the majority of U12-type introns $[33,34]$.

Another example of a universal gene that seems to be missing from the Drosophila genome is sortilin-related receptor LR11 (also known as SorLA), a member of the low-density lipoprotein receptor family. LR11 binds low-density lipoprotein, the major cholesterol-carrying lipoprotein of plasma, and transports it into cells by endocytosis. Human LR11 also regulates trafficking of amyloid precursor protein and its expression is decreased in the brain of Alzheimer's disease patients [35].

Losses of universal multi-copy orthologs

An example is the $\mathrm{Cdc} 7$ kinase and its regulatory subunit $\mathrm{Dbf}_{4}$ implicated in triggering DNA replication in G1 phase through phosphorylation of $\mathrm{Mcm}$ proteins [36]. Cdc7 is essential in yeast in contrast to mice where homozygous null mutants for the $\mathrm{Cdc} 7$ ortholog Nr2c2 show impaired spermatogenesis [37]. Cdc7 is a universal single-copy gene with two fly para- 
logs whereas Dbf4 is present in two copies in humans and opossum. We confirmed the loss of both genes in Tribolium by tBlastn search and phylogenetic analysis (Additional data file 3). Cdc7 is also missing from the current Anopheles annotation but tBlastn searches identified a $\mathrm{Cdc} 7$ candidate in the genome. Dbf4 appears to be missing from the Anopheles and Tetraodon genomes. Interestingly, in yeast an allele of the gene MCM5 (CDC46) has been identified that bypasses the requirement for $\mathrm{CDC}_{7} / \mathrm{DBF}_{4}$ [38]. Although the Tribolium MCM5 ortholog TC_09146 does not feature the same mutation, $\mathrm{P} 86 \mathrm{~L}$, it is conceivable that a similar mutation has rendered $\mathrm{CDC}_{7} / \mathrm{DBF}_{4}$ disposable in Tribolium.

Another example of a loss of an otherwise universal gene is the Tribolium ortholog of human ATP-binding cassette transporter A1 (ABCA1). ABCA1 is a cholesterol efflux transporter and is also required for engulfment of apoptotic cells by macrophages in mice and C. elegans [39,40]. In humans, the turnover of ABCA1 is regulated by Alpha1-syntrophin [41], and both genes encoding these proteins appear to be missing from the beetle genome.

\section{Losses of patchy and insect-specific orthologs}

We observed numerous losses in Diptera, many of which seem to be involved in the ubiquitin cycle, DNA repair (also reported in [31]), actin cytoskeleton and transcription control (Additional data file 4), which may point to substantial rearrangements of the ancestral pathways. An intriguing example is the BRCC complex, a complex with ubiquitin E3 ligase activity known to be involved in DNA repair, cell cycle regulation and homology-directed repair in human that has lost $B R C A 1, R B B P-8$ and $B R C C 3$ in the Dipteran lineage.

An example of insect-specific orthologous groups lost in Diptera are genes associated with oxidoreductase activity, including Aldo/keto reductases and several FAD dependent oxidoreductases; this category of genes was enriched among the 160 Diptera gene losses in a comparative Gene Ontology (GO) analysis with the Tribolium genome (Additional data file 5).

\section{Extreme cases: exclusive insect models of human genes}

At extremes, each novel insect genome sequence uncovers previously invisible orthologous gene relationships to human genes (see [10] for venn diagram that shows how many new orthologous relations are uncovered by the honeybee and beetle genomes). For example, we identified 45 orthologous groups shared between honeybee and at least one vertebrate but lost in the Coleoptera/Diptera ancestor, for example, an ortholog of RAD18 (GB-14468) that is an E3 ubiquitin-protein ligase involved in postreplication repair of UV-damaged DNA.

To complement the initial analysis of the Tribolium genome, we further identified 62 genes that are present in all vertebrates and Tribolium but lost from the other four insect

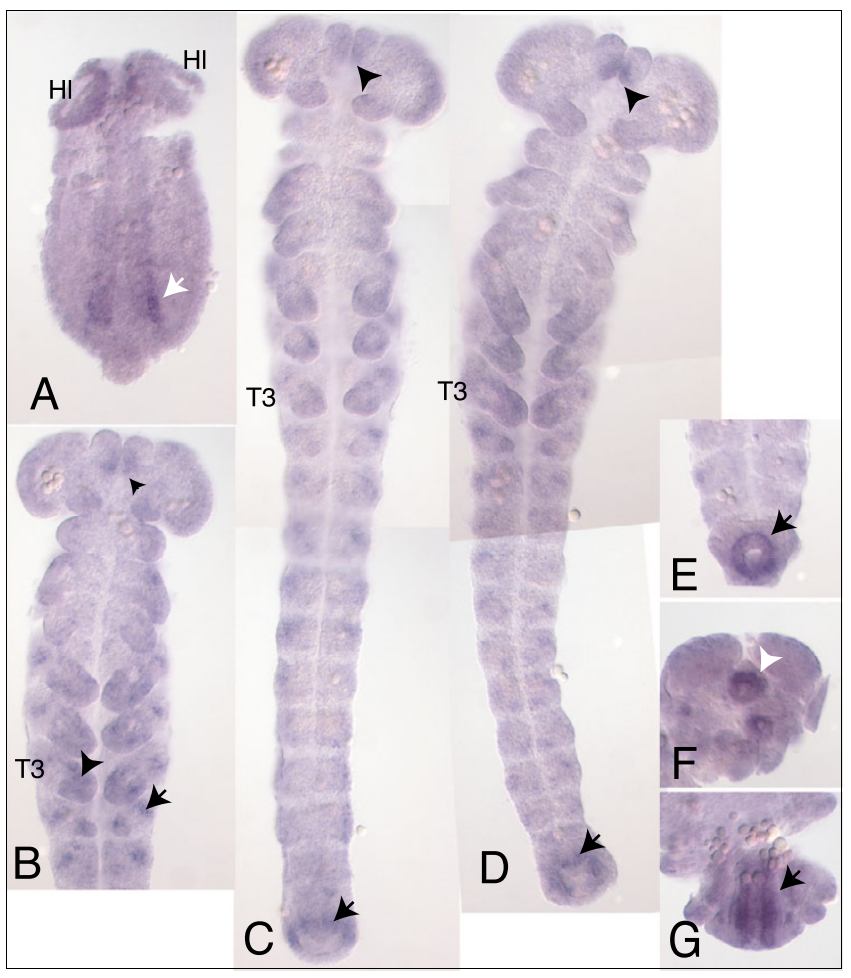

Figure 4

Expression pattern of the F-box gene during embryogenesis of the beetle T. castaneum. (a) F-box gene TC_04309 is initially expressed in the germ rudiment at the rims of the invaginating mesoderm, a position where activated Map-kinase is also seen [76]. Expression is strongest in the posterior (white arrow) and weakens towards the anterior. Ventral view, anterior is up posterior points down. $\mathrm{HI}$, head lobes. (b) Expression is seen as spots in the thoracic legs (arrowhead), at the base of the labral head appendages (small arrow head) and in segmentally repeated spots in the lateral body wall. T3, thoracic segment 3. Only the anterior half of the embryo is shown. (c) At a similar stage as shown in (b) where all body segments are present, the F-box gene is expressed in the anlagen of the hindgut (arrow). (d) When the legs have grown longer, F-box gene expression is extended covering the distal end. As seen in (c, d), expression in the labrum, in the hindgut-primordium and weakly at the lateral sites of the abdominal segments persists. (e) The hindgut has invaginated and grows out, forming a tube where the F-box gene is expressed in its posterior, proximal end around the future posterior gut opening (arrow). (f) At the retracted germ band stage, F-box is expressed around the anterior gut opening (white arrow) that has formed between the head lobes. (g) In the same embryo shown in ( $f$ ), F-box gene expression is seen in the walls of the hindgut (arrow).

genomes. Examples include Yipf3, a natural killer cell-specific antigen expressed during embryonic hematopoiesis in humans, and CENP-S, which in humans is a component of a centromeric protein complex, CENPA-CAD [42], that replaces histones in centromeres. In Tribolium, the orthologs of the other five complex members [42] seem to be absent from the genome, indicating a different mode of action. $C R L F 3$, a cytokine receptor-like factor 3 , has also been lost in all insects but Tribolium, as well as a regulator of the $\mathrm{NF}-\kappa \mathrm{B}$ pathway, $T g f$, which positively regulates I-kappaB kinase. Because of structural and functional similarities in the mode 
of activation between insect and vertebrate NF- $\mathrm{BB} / \mathrm{Rel}$ transcription factors, they are thought to have countered infections in Urbilateria [43]. Another Tribolium gene, TC_04309, absent in all sequenced arthropods, is an ortholog of the human F-box only gene 7 (Fbxo7). The tBlastn search and phylogenetic analysis of $\mathrm{FBXO} 7$ confirmed the lack of clear orthologs in other insects (Additional data files 6 and 7 ). $\mathrm{FBXO}_{7}$ is a component of modular $\mathrm{E}_{3}$ ubiquitin protein ligases called SCFs and functions in phosphorylationdependent ubiquitination [44]. Human FBXO7 was also reported to positively regulate the activity of cyclin D/CDK6 in order to facilitate entry into the cell cycle [45]. In Drosophila, the cyclin D/CDK6 complex stimulates cell growth as well as proliferation [46,47]. Amniotes have two cdk4 orthologs due to a gene duplication (cdk4 and cdk6) and two cyclin D orthologs. Tribolium also encodes a cdk4 ortholog and two cyclin D orthologs, in contrast to the other insects, which have only one cyclin D ortholog (Additional data file 8). It is tempting to speculate that the presence of $\mathrm{FBXO}_{7}$ and a second cyclin D in the beetle are functionally linked. TC_04309 is expressed in limbs and the hindgut of Tribolium embryos (Figure 4). The function of TC_04309 is unknown but, taken together, it is conceivable that it controls cell proliferation in a tissue-specific manner as in mammals.

\section{Robustness of estimates}

Several factors can lead to an overestimation of gene losses: incomplete annotations, genome sequence gaps and the limited sensitivity of protein sequence comparison methods. Reassuringly, our estimates of number of gene losses for Drosophila and human, the two most extensively studied and curated model organisms, are similar to that of automatically annotated species, indicating a fairly good quality of genome annotations and their relative completeness. The chicken genome, however, shows exceptionally high numbers of losses in all categories that are likely to be overestimates due to an incomplete genome sequence that was estimated to be missing $5-10 \%$ of the genes [48], and, therefore, it was not taken into account in the analysis presented above. A closer inspection of 'missing' Tribolium genes from the universal single-copy and insect-specific fraction allowed us to correct about 30 Tribolium genes overlooked by the automatic annotation and some 150 merged genes. Nevertheless, most 'missing' genes were confirmed to be absent from the genome using tBlastn searches, indicating that the Tribolium annotation is nearly complete for evolutionarily conserved genes. Orthology misclassifications can also lead to inflated estimates when orthologous groups are wrongly split up, or to underestimates when several orthologous groups are spuriously pooled together, 'hiding' losses. We compared the results of our analysis with an independently derived and hand curated set of about 100 gene losses in Diptera (Hugh Robertson, personal communication). Detailed phylogenetic examination revealed only two to three cases of likely errors in our high throughput orthology identification pipeline and a few complicated cases that could not be resolved even using phylogenetic methods as the proteins were too short or too divergent.

\section{Discussion}

We present here the quantification of losses of orthologous groups in five vertebrate and five insect genomes. Lineagespecific gene duplications result in multi-copy orthologous groups or fine-grained gene families. Here we focused on complete losses of such gene families (requiring all orthologous genes to be lost in a lineage). Members of an orthologous group are likely to share overlapping functions, and a complete loss of all representatives is more likely to have biological consequences $[49,50]$ than a loss of a specific gene member. The parsimonious interpretation of the losses in the context of the species phylogeny suggests hundreds of gene losses on each branch of the tree. Diptera species lost the most genes, and placentals the least.

We show that the higher numbers of lost genes in insects can be explained by their higher rates of evolution as the loss rate is positively correlated with the molecular rate of evolution for each ortholog category and branch of the phylogeny. Interestingly, losses normalized for evolutionary rate and total number of orthologous groups are similar between insects and vertebrates, even for I and V orthologs. Therefore, one can not exclude that gene losses are mainly driven by neutral evolution $[51,52]$, which should be taken as the null hypothesis until proven otherwise. Our data also suggest that about $20 \%$ of the gene repertoire evolves 8 times faster than the rest. The fact that the overall number of losses of orthologous groups is in agreement with the model of neutral evolution does not, of course, mean that all losses are selectively neutral. In that respect, it is noteworthy that some of the lost genes we discuss, such as $C d c 7 / D b f 4$ or Fboxo7, seem to act as positive regulators.

Several hypotheses have been put forward to explain differences in evolutionary rates across species [53]. A high evolutionary rate might simply reflect differences in mutation rates. The known contributors to the rate of mutations, metabolic rate [54] and generation time [55], are clearly different between dipterans and mammals. In addition, differences in DNA methylation, fidelity of DNA-repair mechanisms or the production of DNA-damaging agents have also been suggested to explain different mutation rates in different species $[53,56]$. We found a number of genes implicated in DNA repair missing from Diptera. Although the functional consequences of these losses in Diptera are unknown, they might contribute to an increased mutation rate. A second hypothesis is that the efficiency of selection against deleterious mutants varies across species, due to differences in effective population size and/or mode of reproduction. Finally, rate variation across lineages could be caused by species-specific differences in the timing and frequency of adaptive evolution. 
Indeed, theoretical models $[57,58]$ have proposed that evolvability is a selectable trait.

\section{Conclusion}

We showed that the gene loss rate correlates well with rates of molecular evolution, explaining the significantly higher number of gene losses in insects. The data also can not reject that gene losses are dominated by neutral evolution.

The hundreds of lost genes we identified along the phylogenetic tree suggest common rearrangements and rewiring of ancient pathways and signaling cascades. Such global approaches are suitable for generating further experimentally testable hypotheses, and will lead to a better understanding of global evolutionary trends and detailed functional differences among lineages.

\section{Materials and methods Orthology classification}

Protein sets were retrieved from Ensembl for Drosophila, Anopheles and all vertebrates as of 4 August 2006. Tribolium and Apis proteins were retrieved from Baylor College of Medecine and Aedes proteins from VectorBase. The assignment to orthologous groups was performed as described earlier $[9,59,60]$. Namely, we retained the longest open reading frame per locus and performed all-against-all comparisons using the Smith-Waterman algorithm as implemented in ParAlign [61] with default parameters. The orthologous groups were then assembled from the best reciprocal hits (BRHs; reciprocally best maching genes in between-genome-comparisons) applying a COG-like [62] procedure to join BRHs across three or more species, going from the best scoring ones until an E-value cut-off of $10^{-6}$, and keeping single BRH pairs only with E-values less than $10^{-10}$. Furthermore, the orthologous groups were expanded by genes that are more similar to each other within a proteome than to any gene in any of the other species, and by very similar copies that share over $97 \%$ sequence identity, which were identified initially using CD-hit [63]. All proteins in a group were required to have aligned regions overlapping by at least 20 residues to avoid the 'domain walking' effect.

\section{Species tree}

A maximum likelihood species tree was calculated using the concatenated multiple alignments of 1,150 orthologs present in exactly one copy in all the organisms studied here. Multiple protein alignments were produced using muscle [64] and confidently aligned regions were extracted using Gblocks [65] with default settings. Individual protein alignments were concatenated into a 336,069 amino acid superalignment that was then subjected to maximum-likelihood analysis using the JTT model $\left(\mathrm{G}_{4}+\mathrm{I}+\mathrm{F}\right)$ as implemented in PhyML [66] and we used Tree-Puzzle [67] to join separate bootstrap analyses. All shown branchings have at least $99 \%$ bootstrap support estimated from 500 replicates.

\section{Quantification of losses and correlation with other traits}

Species radiation dates were taken from the literature: for insects from [18] and for vertebrates from [68,69]. We used MatLab version 7.2 (MathWorks, Natick MA, USA) for statistical analysis and data plotting. Regression lines were required to cross the origin. For each category of orthologs, the slopes of the regression lines for insects and vertebrates were compared based on a Student's $t$-distribution and were found not to be significantly different. Because traits were not normally distributed, we used non-parametric Spearman's correlation coefficients and Mann-Whitney U tests. Chicken data were excluded from graphs and statistical tests (see Discussion).

\section{Manual analysis of case studies}

Selected orthologous groups were examined manually as follows. The absence of Tribolium proteins was verified by screening the Tribolium proteome, genome (assembled and single reads) and expressed sequence tags using the Baylor College of Medicine blast server [70]. All sufficiently similar sequences, including members of other orthologous groups, were aligned using muscle v3.6 [64] with default settings and all positions containing gaps were trimmed from conserved blocks using Gblocks [65]. Phylogenetic trees were constructed using maximum likelihood as implemented in PhyML [66] using the JTT model of amino acid substitution, a gamma distribution of rates over four rate categories and 100 bootstraps.

\section{Pathway mapping and database searches}

We used pathway annotations from the KEGG database [71], mapping genes to Biocarta and co-citation analysis using Webgestalt [29] web interface. For data mining we used Ensembl [72], Swiss-prot/UniProt [73], Flybase [74], the Interactive Fly [22] and Online Mendelian Inheritance in Man [75] annotations.

\section{Abbreviations}

ABCA1, ATP-binding cassette transporter A1; BRH, best reciprocal hit; $\mathrm{CRLF}_{3}$, cytokine receptor-like factor 3; GO, Gene Ontology; I, insect-specific orthologs; MYA, million years ago; N, universal multiple-copy orthologs; $\mathrm{P}$, patchy orthologs; TERT, insect telomerase reverse transcriptase; U, universal single-copy orthologs; V, vertebrate-specific orthologs.

\section{Authors' contributions}

SW and EMZ analyzed the data and wrote the manuscript. EK contributed the orthology data and the species phylogeny. RS and TK contributed experimental characterization of exclu- 
sive Tribolium and honeybee orthologs of human genes. EMZ supervised the project.

\section{Additional data files}

The following additional data are available with the online version of this paper. Additional data file 1 is a graph showing the correlation between the absolute number of lost orthologous groups and and the rate of amino acid substitutions. Additional data files 2 and 3 provide the phylogenetic analysis of SID-1 and $\mathrm{CDC}_{7}$, respectively. Additional data file 4 is a table listing GO analysis of insect-specific orthologous groups lost in all Dipterans. Additional data file 5 is a table listing functionally linked genes coeliminated in the Diptera and Drosophila lineages. Additional file 6 provides the phylogenetic analysis of Fboxo7/cdk4/cyclin D. Additional data file 7 is a figure showing the alignment of Fboxo7 proteins.

\section{Acknowledgements}

We thank Hugh Robertson for sharing unpublished data, Peer Bork for stimulating discussions, and Robert $M$ Waterhouse for help with the manuscript. Swiss National Science Foundation is acknowledged for funding (SNF 3 IOOAO-I I 2588 to EMZ).

\section{References}

I. Samonte RV, Eichler EE: Segmental duplications and the evolution of the primate genome. Nat Rev Genet 2002, 3:65-72.

2. Zhang J: Evolution by gene duplication: an update. Trends Ecol Evol 2003, I 8:292-298.

3. Brosius J: RNAs from all categories generate retrosequences that may be exapted as novel genes or regulatory elements. Gene 1999, 238:115-134.

4. Niimura $Y$, Nei M: Evolutionary dynamics of olfactory and other chemosensory receptor genes in vertebrates. J Human Genet 2006, 5 I:505-517.

5. Gilad Y, Wiebe V, Przeworski M, Lancet D, Paabo S: Loss of olfactory receptor genes coincides with the acquisition of full trichromatic vision in primates. PLoS Biol 2004, 2:E5.

6. Olson MV: When less is more: gene loss as an engine of evolutionary change. Am Human Genet 1999, 64:18-23.

7. Wang X, Grus WE, Zhang J: Gene losses during human origins. PLoS Biol 2006, 4:e52.

8. Greenberg AJ, Moran JR, Fang S, Wu Cl: Adaptive loss of an old duplicated gene during incipient speciation. Mol Biol Evol 2006, 23:40I-4I0.

9. Weinstock GM, Robinson GE, Gibbs RA, Worley KC, Evans JD, Maleszka R, Robertson HM, Weaver DB, Beye M, Bork P, et al.: Insights into social insects from the genome of the honeybee Apis mellifera. Nature 2006, 443:931-949.

10. Consortium TGS: The first genome sequence of a beetle, Tribolium castaneum, a model for insect development and pest biology. Nature in press.

II. Grimaldi D, Engel M: Evolution of the Insects Cambridge: Cambridge University Press; 2005.

12. Demuth JP, Bie TD, Stajich JE, Cristianini N, Hahn MW: The evolution of Mammalian gene families. PLOS ONE 2006, I:e85.

13. Blomme T, Vandepoele K, De Bodt S, Simillion C, Maere S, Van de Peer $Y$ : The gain and loss of genes during 600 million years of vertebrate evolution. Genome Biol 2006, 7:R43.

14. Koonin EV, Fedorova ND, Jackson JD, Jacobs AR, Krylov DM, Makarova KS, Mazumder R, Mekhedov SL, Nikolskaya AN, Rao BS, et al.: A comprehensive evolutionary classification of proteins encoded in complete eukaryotic genomes. Genome Biol 2004, 5:R7.

15. Hughes AL, Friedman R: Differential loss of ancestral gene families as a source of genomic divergence in animals. Proc Biol Sci 2004, 27 I(Suppl 3):SI07-109.
16. Wang $Y$, Jorda M, Jones PL, Maleszka R, Ling X, Robertson HM, Mizzen CA, Peinado MA, Robinson GE: Functional CpG methylation system in a social insect. Science 2006, 3 I 4:645-647.

17. Rubin EB, Shemesh Y, Cohen M, Elgavish S, Robertson HM, Bloch G: Molecular and phylogenetic analyses reveal mammalian-like clockwork in the honey bee (Apis mellifera) and shed new light on the molecular evolution of the circadian clock. Genome Res 2006, 16:1352-1365.

I8. Zdobnov EM, Mering C, Letunic I, Bork P: Consistency of genomebased methods in measuring Metazoan evolution. FEBS Lett 2005, 579:3355-336I.

19. Savard J, Tautz D, Richards S, Weinstock GM, Gibbs RA, Werren JH, Tettelin H, Lercher MJ: Phylogenomic analysis reveals bees and wasps (Hymenoptera) at the base of the radiation of Holometabolous insects. Genome Res 2006, I 6:I334.

20. Glise B, Miller CA, Crozatier M, Halbisen MA, Wise S, Olson DJ, Vincent A, Blair SS: Shifted, the Drosophila ortholog of Wnt inhibitory factor-I, controls the distribution and movement of Hedgehog. Dev Cell 2005, 8:255-266.

21. Kernan M, Cowan D, Zuker C: Genetic dissection of mechanosensory transduction: mechanoreception-defective mutations of Drosophila. Neuron 1994, I 2: I I95- I 206.

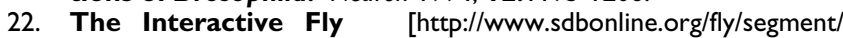
shifted4.htm]

23. Zdobnov EM, von Mering C, Letunic I, Torrents D, Suyama M, Copley RR, Christophides GK, Thomasova D, Holt RA, Subramanian GM, et al.: Comparative genome and proteome analysis of Anopheles gambiae and Drosophila melanogaster. Science 2002, 298:149-I59.

24. Robertson HM, Gordon KHJ: Canonical TTAGG-repeat telomeres and telomerase in the honey bee, Apis mellifera. Genome Res 2006, 1 6: I345.

25. Pellegrini M, Marcotte EM, Thompson MJ, Eisenberg D, Yeates TO: Assigning protein functions by comparative genome analysis: Protein phylogenetic profiles. Proc Natl Acad Sci USA I999, 96:4285-4288.

26. Aravind L, Watanabe H, Lipman DJ, Koonin EV: Lineage-specific loss and divergence of functionally linked genes in eukaryotes. Proc Natl Acad Sci USA 2000, 97: I I 3 | 9 - I I 324.

27. Barker D, Pagel M: Predicting functional gene links from phylogenetic-statistical analyses of whole genomes. Plos Comput Biol 2005, I:24-31.

28. Stark C, Breitkreutz BJ, Reguly T, Boucher L, Breitkreutz A, Tyers M: BioGRID: a general repository for interaction datasets. Nucleic Acids Res 2006, 34:D535-539.

29. Zhang B, Kirov S, Snoddy J: WebGestalt: an integrated system for exploring gene sets in various biological contexts. Nucleic Acids Res 2005, 33:W74I-W748.

30. Will CL, Luhrmann R: Splicing of a rare class of introns by the U I2-dependent spliceosome. Biol Chem 2005, 386:713-724.

31. Adams MD, Celniker SE, Holt RA, Evans CA, Gocayne JD, Amanatides PG, Scherer SE, Li PW, Hoskins RA, Galle RF: The Genome Sequence of Drosophila melanogaster. Science 2000, 287:2 I 85.

32. Schneider C, Will CL, Brosius J, Frilander MJ, Luhrmann R: Identification of an evolutionarily divergent $U$ I I small nuclear ribonucleoprotein particle in Drosophila. Proc Natl Acad Sci USA 2004, I 01 :9584-9589.

33. Mount SM, Gotea V, Lin CF, Hernandez K, Makalowski W: Spliceosomal small nuclear RNA genes in I I insect genomes. RNA 2007, I 3:5-14.

34. Sheth N, Roca X, Hastings ML, Roeder T, Krainer AR, Sachidanandam $\mathrm{R}$ : Comprehensive splice-site analysis using comparative genomics. Nucleic Acids Res 2006, 34:3955-3967.

35. Spoelgen R, von Arnim CA, Thomas AV, Peltan ID, Koker M, Deng A Irizarry MC, Andersen OM, Willnow TE, Hyman BT: Interaction of the cytosolic domains of sorLA/LRI I with the amyloid precursor protein (APP) and beta-secretase beta-site APP. cleaving enzyme. J Neurosci 2006, 26:418-428.

36. Bell SP, Dutta A: DNA replication in eukaryotic cells. Annu Rev Biochem 2002, 71:333-374.

37. Mu X, Lee YF, Liu NC, Chen YT, Kim E, Shyr CR, Chang C: Targeted inactivation of testicular nuclear orphan receptor 4 delays and disrupts late meiotic prophase and subsequent meiotic divisions of spermatogenesis. Mol Cell Biol 2003, 24:5887-5899.

38. Hardy CFJ, Dryga O, Seematter S, Pahl PMB, Sclafani RA: mcm5/ cdc46-bob I bypasses the requirement for the $S$ phase activator Cdc7p. Proc Natl Acad Sci USA 1997, 94:3। II-3I 55. 
39. Hamon Y, Broccardo C, Chambenoit O, Luciani MF, Toti F, Chaslin S, Freyssinet JM, Devaux PF, McNeish J, Marguet D: ABCI promotes engulfment of apoptotic cells and transbilayer redistribution of phosphatidylserine. Nat Cell Biol 2000, 2:399-406.

40. Sheps JA, Ralph S, Zhao ZY, Baillie DL, Ling V: The ABC transporter gene family of Caenorhabditis elegans has implications for the evolutionary dynamics of multidrug resistance in eukaryotes. Genome Biol 2004, 5:R I5.

41. Munehira Y, Ohnishi T, Kawamoto S, Furuya A, Shitara K, Imamura M, Yokota T, Takeda S, Amachi T, Matsuo M: \{alpha\} I-Syntrophin modulates turnover of ABCAI. Biol Chem 2004, 279: I509I-I5095.

42. Foltz DR, Jansen LE, Black BE, Bailey AO, Yates JR 3rd, Cleveland DW: The human CENP-A centromeric nucleosome-associated complex. Nat Cell Biol 2006, 8:458-469.

43. Hoffmann JA, Reichhart JM: Drosophila innate immunity: an evolutionary perspective. Nat Immunol 2002, 3:121-126.

44. Hsu JM, Lee YC, Yu CT, Huang CY: Fbx7 functions in the SCF complex regulating Cdkl-cyclin B-phosphorylated hepatoma up-regulated protein (HURP) proteolysis by a proline-rich region. Biol Chem 2004, 279:32592-32602.

45. Laman H, Funes JM, Ye H, Henderson S, Galinanes-Garcia L, Hara E, Knowles $\mathrm{P}$, McDonald N, Boshoff C: Transforming activity of Fbxo7 is mediated specifically through regulation of cyclin D/ cdk6. EMBO J 2005, 24:3 I04-3II6.

46. Datar SA, Jacobs HW, de la Cruz AFA, Lehner CF, Edgar BA: The Drosophila cyclin D-cdk4 complex promotes cellular growth. $E M B O$ ] 2000, 19:4543-4554.

47. Meyer CA, Jacobs HW, Datar SA, Du W, Edgar BA, Lehner CF: Drosophila Cdk4 is required for normal growth and is dispensable for cell cycle progression. EMBO j 2000, 19:4533-4542.

48. Hillier LW, Miller W, Birney E, Warren W, Hardison RC, Ponting CP, Bork P, Burt DW, Groenen MAM, Delany ME, et al.: Sequence and comparative analysis of the chicken genome provide unique perspectives on vertebrate evolution. Nature 2004, 432:695-716.

49. Kamath RS, Fraser AG, Dong Y, Poulin G, Durbin R, Gotta M, Kanapin A, Le Bot N, Moreno S, Sohrmann M: Systematic functional analysis of the Caenorhabditis elegans genome using RNAi. Nature 2003, 421:23I-237.

50. Gu Z, Steinmetz LM, Gu X, Scharfe C, Davis RW, Li WH: Role of duplicate genes in genetic robustness against null mutations. Nature 2003, 42 I:63-66.

5I. Kimura M, Takahata N: Selective constraint in protein polymorphism: study of the effectively neutral mutation model by using an improved pseudosampling method. Proc Natl Acad Sci USA 1983, 80: 1048-1052.

52. Ohta $\mathrm{T}$ : Slightly deleterious mutant substitutions in evolution. Nature 1973, 246:96-98.

53. Pál C, Papp B, Lercher MJ: An integrated view of protein evolution. Nat Rev Genet 2006, 7:337-348.

54. Rand DM, Kann LM: Excess amino acid polymorphism in mitochondrial DNA: contrasts among genes from Drosophila, mice, and humans. Mol Biol Evol 1996, 13:735-748.

55. Kohne DE: Evolution of higher-organism DNA. Quart Rev Biophys 1970, 3:327-375.

56. Kumar S: Molecular clocks: four decades of evolution. Nat Rev Genet 2005, 6:654-662.

57. Wagner A: Does evolutionary plasticity evolve? Evolution 1996, 50:1008-1023.

58. Earl DJ, Deem MW: Evolvability is a selectable trait. Proc Natl Acad Sci USA 2004, 101: I 1531-1 I 536.

59. Kriventseva EV, Rahman N, Espinosa O, Zbodnov EM: OrthoDB: the hierarchical catalog of eukaryotic orthologs. Nucleic Acids Res 2007. doi: I0.1093/nar/gkm845.

60. Zdobnov EM, Bork P: Quantification of insect genome divergence. Trends Genet 2007, 23:16-20.

61. Saebo PE, Andersen SM, Myrseth J, Laerdahl JK, Rognes T: PARALIGN: rapid and sensitive sequence similarity searches powered by parallel computing technology. Nucleic Acids Res 2005, 33:W535-539.

62. Tatusov RL, Koonin EV, Lipman DJ: A genomic perspective on protein families. Science 1997, 278:631-637.

63. Li W, Godzik A: Cd-hit: a fast program for clustering and comparing large sets of protein or nucleotide sequences. Bioinformatics 2006, 22: 1658-1659.

64. Edgar RC: MUSCLE: multiple sequence alignment with high accuracy and high throughput. Nucleic Acids Res 2004,
32: $1792-1797$.

65. Castresana J: Selection of conserved blocks from multiple alignments for their use in phylogenetic analysis. Mol Biol Evol 2000, 17:540-552.

66. Guindon S, Gascuel O: A simple, fast, and accurate algorithm to estimate large phylogenies by maximum likelihood. Syst Biol 2003, 52:696-704.

67. Schmidt HA, Strimmer K, Vingron M, von Haeseler A: TREE-PUZZLE: maximum likelihood phylogenetic analysis using quartets and parallel computing. Bioinformatics 2002, 18:502-504.

68. Kumar S, Hedges SB: A molecular timescale for vertebrate evolution. Nature 1998, 392:917-920.

69. Hedges SB: The origin and evolution of model organisms. Nat Rev Genet 2002, 3:838-849.

70. Baylor College of Medicine Blast Server: T. castaneum [http:/ /www.hgsc.bcm.tmc.edu/blast/blast.cgi?organism=Tcastaneum]

7I. Kanehisa M, Goto S: KEGG: Kyoto Encyclopedia of Genes and Genomes. Nucleic Acids Res 2000, 28:27-30.

72. Hubbard TJP, Aken BL, Beal K, Ballester B, Caccamo M, Chen Y, Clarke L, Coates G, Cunningham F, Cutts T, et al.: Ensembl 2007. Nucleic Acids Res 2007, 35:D610-617.

73. Bairoch A, Bougueleret L, Altairac S, Amendolia $V$, Auchincloss A, Puy GA, Axelsen K, Baratin D, Blatter MC, Boeckmann B, et al:: The universal protein resource (UniProt). Nucleic Acids Res 2007, 35:D193-DI97.

74. Crosby MA, Goodman JL, Strelets VB, Zhang PL, Gelbart WM: FlyBase: genomes by the dozen. Nucleic Acids Res 2007, 35:D486-D49I.

75. Online Mendelian Inheritance in Man [http:// www.ncbi.nlm.nih.gov/omim]

76. Schoppmeier M, Schröder R: Maternal torso signaling controls body axis elongation in a short germ insect. Curr Biol 2005 , 15:2|3|-2|36. 